\title{
Male Manipulation of Female Receptivity in Sagebrush Crickets
}

\section{Authors}

Carie B. Weddle, Rachel L. Avery, Pamela L. Brady, John K. Sakaluk, Jason H. Trullinger, and Scott K. Sakaluk 


\title{
Male Manipulation of Female ReCePtivity IN SAGEBRUSH CRICKETS
}

\author{
CARIE B. WEDDLE $\uparrow$ RACHEL L. AVERY $\uparrow$ PAMELA L. BRADY \\ JOHN K. SAKALUK \ JASON H. TRULLINGER \ SCOTT K. SAKALUK \\ BEHAVIOR, ECOLOGY, EVOLUTION AND SYSTEMATICS SECTION \\ DEPARTMENT OF BIOLOGICAL SCIENCES \\ ILLINOIS STATE UNIVERSITY $\uparrow$ NORMAL
}

\section{$\uparrow \quad$ ABSTRACT}

Male sagebrush crickets, Cyphoderris strepitans, offer an unusual nuptial food gift to females during copulation: females are permitted to feed on the hind wings of males and ingest hemolymph that flows from the resulting wounds. A previous study of this species showed that females experimentally precluded from wing feeding during initial copulations were more receptive to subsequent matings than females allowed to obtain a full blood meal during initial copulations (Johnson et al. 1999). The present study was designed to test the hypothesis that hormonal substances contained in the hemolymph of males and ingested by females during copulation function to decrease female receptivity to further matings, and thereby constitute male manipulation. We tested this hypothesis by allowing females to ingest one of the following substances prior to experimental pairings: adult male hemolymph, adult female hemolymph, or insect Ringer's solution. Experimental females were given the opportunity to mate 24 hours after treatments were established and latency to mating was used as a measure of female receptivity. There was no significant difference in latency to mating across treatments. The lack of a difference could be due to a decay in the activity of putative hormonal substances over the 24-hour period separating hemolymph delivery and mating trials. In addition, the simultaneous act of wing-feeding combined with
\end{abstract}

intitial copulation may provide a behavioral primer that triggers any effect on female receptivity.

\section{$\uparrow \quad$ INTRODUCTION}

Sexual conflicts between males and females may arise in decisions concerning the use of sperm for fertilizations, particularly when females mate with more than one male. While it is always in a male's best interests to have all of his sperm utilized, females may benefit by being selective of potential sires for their offspring. In many insects, females have more control over reproduction than do males, because they not only control the transfer of sperm during copulation, but may also exhibit postcopulatory mechanisms that determine whether stored sperm are actually used in fertilizations (Thornhill and Alcock 1983; Sakaluk 1984). Such cryptic female choice is in direct evolutionary conflict with the reproductive strategy of males, whose fitness is maximized only when fertilizations result from their gametic investment.

Counter strategies in males to ensure fertilization include mate guarding, mate sequestering, multiple or forced copulation, or the use of specific anti-receptivity devices (e.g. mating plugs) or anti-receptivity substances (Andersson and Iwasa 1996; Sakaluk et al. 1995). The use of antireceptivity substances such as hormones, in which chemical substances are transferred from the male to 
the female during copulation, has been reported for several species of insects (Eberhard 1996). Juvenile hormone has been shown to be an important factor in the physiological control of female receptivity (Englemann 1970). Shirk et al. (1980) showed that male Cecropia silkmoths transfer large quantities of juvenile hormone to females in their ejaculate during copulation. Exogenous application of juvenile hormone to female leafroller moths (Platynota stultana) induced a switch from virgin to mated (nonreceptive) behavior, and consequently, females ceased production of their sexual-signaling pheromones (Webster and Cardé 1984).

Male sagebrush crickets, Cyphoderris strepitans, offer an unusual nuptial food gift to females during copulation: females are permitted to feed on the fleshy hind wings of males and ingest hemolymph that flows from the wounds they inflict. The wounds are not fatal and females usually only consume a portion of the hind wings, so that males are not precluded from mating again (Dodson et al. 1983). In a recent study by Johnson et al. (1999), female $C$. strepitans were given the opportunity to mate with males subjected to one of two experimental manipulations: 1) males with intact hind wings in which copulatory wing feeding was possible and 2) males whose wings had been surgically removed so that no copulatory wing feeding was possible. Females were then given the opportunity to remate with untreated, virgin males (hind wings intact). The results of this study revealed that females who were prevented from feeding on the wings of their initial mate remated significantly sooner than females who were free to feed during their initial mating.

Johnson et al. (1999) concluded that differential nutrient acquisition via copulatory feeding may give rise to cryptic mate choice in which females use deficiencies in the original male (i.e. lack of nuptial food gift) as a cue to seek additional matings from males who may offer a greater nutritional investment. Alternatively, the results could be taken as evidence of male manipulation, in which females allowed to wing feed during initial matings ingest specific chemical substances contained in male hemolymph that act to decrease their receptivity to further matings (Johnson et al. 1999).

The current study was designed to test the hypothesis that hormonal substances contained in the hemolymph of males and ingested by females during copulation may function as a manipulative strategy of males to decrease female receptivity to further matings. To test this hypothesis, females captured early in the breeding season (May-June 1999) were fed either hemolymph obtained from adult males, hemolymph obtained from adult females, or insect Ringer's solution. The "male manipulation" hypothesis predicts that ingestion of adult male hemolymph should increase female latency to copulation relative to that of females who are allowed to ingest the hemolymph of adult females or insect Ringer's solution.

\section{METHODS}

The study was conducted at the University of Wyoming-National Park Service Research Center, where previous studies of this species have been conducted (Snedden and Sakaluk 1992; Eggert and Sakaluk 1994; Sakaluk et al. 1995). Early in the breeding season (mid May), adult males and adult females were collected from several populations within Grand Teton National Park and transported to the field station. Experimental subjects were maintained at the field station according to standard procedures (Snedden and Sakaluk 1992; Eggert and Sakaluk 1994). All males used in the experiment were virgins, as indicated by intact hind wings. The mating status of females was unknown.

Females were randomly assigned to one of three experimental treatments:

1) Females allowed to consume $20 \mu$ l of hemolymph obtained from adult males $(\mathrm{N}=16)$; 2) Females allowed to consume $20 \mu \mathrm{l}$ of hemolymph obtained from adult females $(\mathrm{N}=16)$; 3) Females allowed to consume $20 \mu$ l of insect Ringer's solution $(N=17)$.

Females were assigned to experimental treatments the evening following their capture and paired with virgin males in mating trials 24 hours after treatment delivery. Experimental pairs were placed into specially constructed Plexiglas viewing chambers divided into two equal compartments $(10 \mathrm{x}$ $6.8 \times 4.4 \mathrm{~cm}$ ). Each compartment was equipped with a calling perch in the form of a short stick. Pairs were established early in the evening when the crickets normally become sexually active and mating behaviors were monitored over the next 12 hours using time-lapse video recording and direct observation. No food or water was provided during mating trials.

Upon review of the video recordings, the following measures of female receptivity were recorded relative to the start of the trial: 
1) Time at which females first mounted males and total number of mounts;

2) Time at which successful copulation occurred (indicated by transfer of the spermatophore).

An ANOVA was used to compare latency of females to mount males across treatments. Nonparametric failure-time analysis was used in comparisons of time to mating. Failure-time analysis provides a method for accommodating censored data, such as observations in which an event may not have occurred before the end of the study (Fox 1993). Data such as these are often mistakenly omitted from subsequent analysis, which can lead to highly biased comparisons (Fox 1993).

Hemolymph was extracted from males by severing one of the hind wings and drawing hemolymph from the wound using a microhematocrit capillary tube. Extraction of hemolymph from adult females involved making a small incision in the cuticle of the pronotum and then, using the same technique, suctioning hemolymph from the wound once it began to bleed. Wounds caused by these procedures normally heal very rapidly and the insects were observed to recover fully from their operations. None of the males used to obtain hemolymph were used in mating trials. All treatments were presented on a small piece of iceberg lettuce and time of treatment ingestion was recorded using time-lapse video photography.

\section{$\uparrow \quad$ RESUlTS}

The results of our study show no significant difference in female receptivity across treatments. Mean latency to successful copulation (Fig. 1) was not significantly different across treatments (Failuretime analysis: $\chi^{2}=0.273, P=0.872$ ). Total number of female mounts (Fig. 2) also did not differ significantly across treatments (Kruskal-Wallis test: $\mathrm{H}_{2}=2.732, \mathrm{P}=0.256$ ). For all treatments, latency to mating was not related to time of treatment consumption (all $\mathrm{P}>0.05$ ).

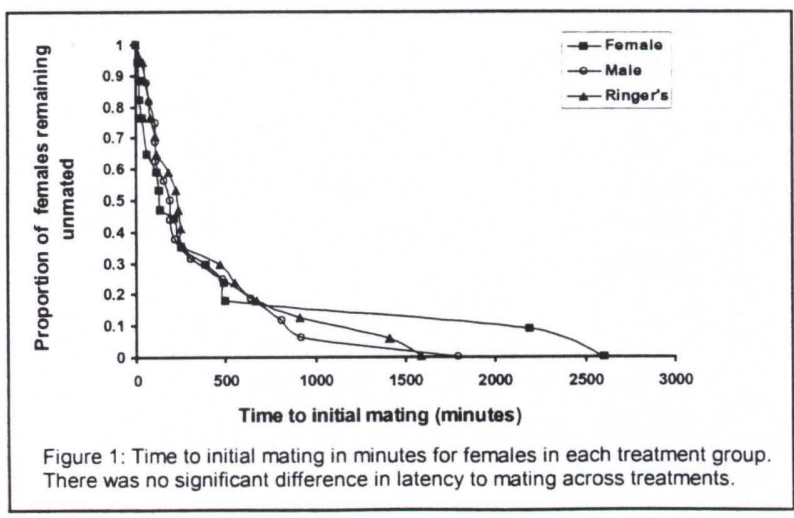

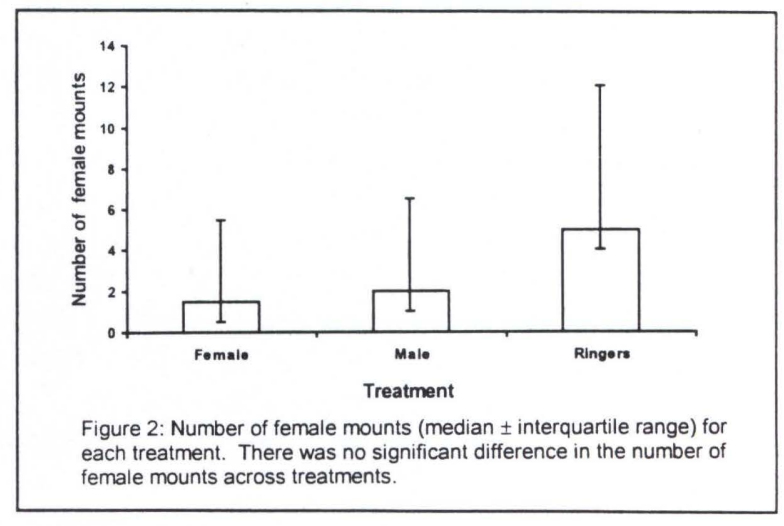

\section{$\downarrow \quad$ DISCUSSION}

Female receptivity was not affected by ingestion of male hemolymph prior to mating. These results are inconsistent with the male manipulation hypothesis and in apparent contradiction of the results of Johnson et al. (1999). A possible factor not accounted for in our experimental protocol is the possibility that putative hormonal substances in male hemolymph may have degraded over the 24-hour period between treatment presentation and subsequent mating trials. However, no relationship was found between time of treatment ingestion and latency to mating.

Alternatively, it may be that any effect of male hemolymph on the subsequent receptivity of females is primed by copulation per se, or the receipt of sperm or other substances in males' ejaculates. In the study by Johnson et al. (1999), females were first mated to a de-winged male before they were given the opportunity to mate with an unmanipulated male. In May-June 2000, we plan to test this possibility by establishing treatment regimes simultaneously with initial copulations. Females will be mated with a virgin male whose hind wings have been removed to prevent wing feeding, and treatments will be offered immediately after successful copulation. Subsequent mating trials will be established 1 hour after treatment ingestion to control for any time-limited effectiveness of hemolymph consumption on female receptivity.

\section{Literature Cited}

Andersson, M. \& Iwasa, Y. 1996. Sexual selection. Trends in Ecology \& Evolution, 11: 53-58. 
Dodson, G. N., Morris, G. K., \& Gwynne, D. T. 1983. Mating behavior in the primitive orthopteran genus Cyphoderris (Haglidae). In: Orthopteran mating systems: sexual competition in a diverse group of insects (D. T. Gwynne \& G. K. Morris, eds.), pp. 305318. Boulder, Colorado: Westview Press.

Eberhard. W. G. 1996. Female control: sexual selection by cryptic female choice. Princeton, New Jersey: Princeton University Press.

Eggert, A-K, Sakaluk, S. K. 1994. Sexual cannibalism and its relation to male mating success in sagebrush crickets, Cyphoderris strepitans (Haglidae: Orthoptera).Animal Behaviour, 47: 1171-1177.

Englemann, F. 1970. The physiology of insect reproduction. Elmsford, New York: Pergamon Press.

Fox, G. A. 1993. Failure-time analysis: emergence, flowering, survivorship and other waiting times. In: Design and analysis of ecological experiments (S. M. Scheiner \& J. Gurevitch), pp. 253-289. New York: Chapman \& Hall.

Johnson, J. C., Ivy, T. M. \& Sakaluk, S. K. 1999. Female remating propensity contingent on sexual cannibalism in sagebrush crickets, Cyphoderris strepitans: a mechanism of cryptic female choice. Behavioral Ecology, 10: 227-233.
Sakaluk, S. K. 1984. Male crickets feed females to ensure complete sperm transfer. Science, 223: 609-610.

Sakaluk, S. K. Bangert, P. J., Eggert, A-K, Gack, C., \& Swanson, L. V. 1995. The gin trap as a device facilitating coercive mating in sagebrush crickets. Proceedings of the Royal Society of London, B, 261: 65-71.

Shirk, P. D., Bhaskaran, G. \& Röller, H. 1980. The transfer of juvenile hormone from male to female during mating in the Cecropia silkmoth. Experientia, 36: 682-683.

Snedden, W. A. \& Sakaluk, S. K. 1992. Acoustical signaling and its relation to male mating success in sagebrush crickets. Animal Behaviour, 44: 633-639.

Thornhill, R., \& Alcock, J. 1983. The evolution of insect mating systems. Cambridge, MA: Harvard University Press.

Webster, R. P. \& Cardé, R. T. 1984. The effects of mating, exogenous juvenile hormone and a juvenile hormone analogue on pheromone titre, calling and oviposition in the omnivorous leafroller moth (Platynota stultana). Journal of Insect Physiology, 30: 113-188. 